\title{
Nadir airborne lidar observations of deep aerosol layers
}

\author{
F. Marenco \\ Observational Based Research, Met Office, Exeter, UK \\ Correspondence to: F. Marenco (franco.marenco@metoffice.gov.uk) \\ Received: 4 April 2013 - Published in Atmos. Meas. Tech. Discuss.: 14 May 2013 \\ Revised: 10 July 2013 - Accepted: 12 July 2013 - Published: 15 August 2013
}

\begin{abstract}
The observation of deep and optically thick aerosol layers by a nadir-pointing lidar poses a challenge in terms of the signal inversion into a geophysically meaningful quantity such as extinction coefficient. A far-end reference molecular layer will usually be unavailable if the observed layer is near the surface, and using a near-end reference results in an unstable mathematical solution. In this paper, it is demonstrated that a far-end reference, taken within the aerosol layer, yields a better solution, and that the influence of the reference reduces strongly when coming inward, so that $1-2 \mathrm{~km}$ above reference the solution can be trusted. A method is developed to set the reference using the assumption of a well-mixed layer near the surface, and its effect is tested on data collected during recent aircraft-based campaigns. The method is also tested on simulated profiles in order to verify its limits and accuracy. The assumption of a well-mixed layer can be relaxed if one is able to set the reference well within a layer rather than at its boundaries.
\end{abstract}

\section{Introduction}

Several papers have appeared in the last decades discussing the solution to the lidar equation for an elastic-backscattering system (see e.g. Fernald et al., 1972; Fernald, 1984; Klett, 1985; Kovalev, 1993; Takamura et al., 1994; Marenco et al., 1997; Young and Vaughan, 2009; Marenco and Hogan, 2011; Donovan and Apituley, 2013). It is generally accepted that the inversion of lidar signals is an ill-constrained task due to the fact that multiple mathematical solutions exist for any given signal profile, so that assumptions need to be made in an intelligent way. Most times, the Fernald-Klett solution is used (i.e. an extinction-to-backscatter ratio (lidar ratio) is assumed, based on aerosol type), and moreover a reference value of the extinction coefficient is set a priori for a chosen layer (Fernald, 1984; Klett, 1985). This is known to be stable only if the reference value is set at the far end of the lidar profile. Alternatively, two reference values can be set, one below and one above an aerosol layer, and in that case the value of the lidar ratio need not be assumed (Di Girolamo et al., 1994). A detailed discussion of the options available for the inversion of lidar signals has been given in the introduction of a previous paper, and therefore it will not be repeated here (Marenco and Hogan, 2011).

However, so far the idea has been that in the absence of independent measurements from a different instrument, the reference value is to be set for a Rayleigh scattering (molecular) layer. Most lidar systems are placed at the ground and point to the zenith, so that in general one can expect to find aerosol-free layers in the far field. This paper deals instead with the case of a nadir-pointing lidar, which looks at the atmosphere from above: in general, therefore, one will not find an aerosol-free layer at the far end, but rather at the near end. This poses a new challenge in terms of the reference values that can be set for signal inversion, and as is shown here in certain circumstances the best approach is to set a reference value within an aerosol layer.

This research is motivated by the important datasets that have been collected on board the Facility for Airborne Atmospheric Measurements (FAAM, http://www.faam.ac.uk) BAe-146 research aircraft during recent campaigns. In April and June 2011 and in June 2012, the Fennec campaign (The Saharan Climate System) performed 35 research flights investigating the Sahara heat low region, and the lidar observed many deep and optically thick mineral dust layers (Ryder et al., 2013). In September and October 2012, the South AMerican Biomass Burning Analysis (SAMBBA) campaign was carried out in Brazil, and several observations of smoke from biomass burning were made during 20 science flights (Angelo, 2012). 
In the future, aircraft and space-borne research will definitely evolve into using high spectral resolution lidar (Ingmann, 2004; Burton et al., 2013; Groß et al., 2013). This better instrument allows extinction and backscatter to be measured separately (as does Raman lidar; see e.g. Ferrare et al., 1998), and thus simplifies the work of the data analyst. A well-characterised and calibrated instrument, having high spectral resolution capability and depolarisation, can really improve what can be achieved with lidar and speed up retrievals. Unfortunately, at the moment this is unavailable to us. We must look for methods for working with simple backscattering data, and a heavy human interaction remains necessary to review assumptions and constraints on a caseby-case basis.

\section{Inversion of lidar signals and associated uncertainties}

\subsection{Outward and inward Fernald-Klett solution}

The lidar system used in the Fennec and SAMBBA campaigns is an ALS450 manufactured by Leosphere, mounted on the aircraft in a nadir-viewing geometry, and with an operating wavelength of $355 \mathrm{~nm}$ (Marenco et al., 2011). Figure $1 \mathrm{a}$ and $\mathrm{b}$ show the vertical profiles of the lidar rangecorrected signal for a typical Fennec and a typical SAMBBA flight. In Fig. 1a, the qualitative analysis of the profile reveals an aerosol-free region above $6 \mathrm{~km}$; the increased signal between 5.4 and $6 \mathrm{~km}$ obviously indicates a dust layer, and so does the steady decrease of signal beyond that range, explained in terms of aerosol extinction of the signal. As the latter decrease shows a homogeneous slope, which is very different from the Rayleigh scattering slope, we can expect that the aerosol extinction coefficient through the layer is more or less constant all the way to the ground. Similarly, in Fig. 1b an aerosol-free layer is observed above $4 \mathrm{~km}$, with a few smoke layers below; the main layer is found between 1.3 and $3.3 \mathrm{~km}$, and a cleaner (but not aerosol-free) layer is found below, where the signal slope is less steep.

If one wants to invert the lidar signals with the FernaldKlett method, one has to set an a priori lidar ratio. In this paper, a lidar ratio of $60 \mathrm{sr}$ for the mineral dust case (first profile) is assumed, and $75 \mathrm{sr}$ is assumed for smoke (second profile); these values are inferred from the literature (Groß et al., 2012, Fig. 14; for dust, see also Amiridis et al., 2013). Besides the lidar ratio, the Fernald-Klett method requires also the a priori setting of a known layer in terms of optical properties; in the traditional application of this method, the latter is a molecular layer. In this scenario, this is only possible above the aerosols (i.e. in the near field and with outward integration), and the outcome of the inversion diverges rapidly, as shown in Fig. 1c and d. This illustrates the instability of the outward Fernald-Klett inversion, which was already discussed many years ago by Fernald (1984).
The inward Fernald-Klett inversion is much more stable, and this is illustrated in Fig. 1e and f. The difficulty here is setting the far-end reference value, i.e. for the nadir geometries considered here, the a priori aerosol extinction coefficient near the ground. In this example, a few representative values with no objective criterion have been picked: 0,75 , 150 and $250 \mathrm{Mm}^{-1}$ for the $0.5-1 \mathrm{~km}$ altitude range. The result is that as we move inward from the reference height $Z_{\mathrm{c}}$, the retrieved aerosol extinction coefficient becomes more and more independent of the reference value. The consequence is a paradox: a wrong value at the far end is better than a correct value at the near end. In these examples, it can be confirmed that $1-1.5 \mathrm{~km}$ above the reference height the influence of the reference value is sufficiently small to be acceptable, and that it becomes irrelevant further up. Of course, there remains a large uncertainty on the magnitude of the aerosol extinction coefficient below $\sim 2 \mathrm{~km}$. The aim of this paper is to explore the different possibilities available to improve the extinction profile retrieval.

\subsection{An improved slope method}

In the previous discussion, basic qualitative information on the aerosol layers was derived, before applying an inversion scheme, by examining the vertical profile of the rangecorrected signal in Fig. 1a and b, and more specifically its slope. This reflects the fact that the rate at which the lidar signal decreases with increasing range is directly related to the turbidity of the atmosphere. As a matter of fact, the slope of lidar signals has been exploited to derive the extinction coefficient since the beginning of lidar research (Collis, 1966), and an interesting study on this type of approach has been carried out by Kunz and de Leeuw (1993).

Strictly speaking, the slope method as it is treated in the above references is limited to single-component homogeneous atmospheres, i.e. where the extinction coefficient is constant with range, a scenario that will seldom apply to the vertical profiling of aerosols at optical wavelengths. The interesting point, however, is that it is neither required to have a reference value, nor to assume a lidar ratio. We shall here write the equations for the more general case (inhomogeneous two-component atmosphere) in order to better frame the terms of this approximation, and see in which cases the slope method could be used. We start from the singlescattering lidar equation:

$$
\begin{aligned}
P & =K\left(\beta_{\mathrm{m}}+\beta_{\mathrm{a}}\right) e^{-2 \int_{0}^{R}\left(\alpha_{\mathrm{m}}+\alpha_{\mathrm{a}}\right) \mathrm{d} R} \\
& =K \beta_{\mathrm{m}} \mathrm{BR} e^{-2 \int_{0}^{R}\left(\alpha_{\mathrm{m}}+\alpha_{\mathrm{a}}\right) \mathrm{d} R},
\end{aligned}
$$

where $P(R)$ is the range-corrected lidar signal, $\beta_{i}(R)$ the backscattering coefficient, $\alpha_{i}(R)$ the extinction coefficient, $\mathrm{BR}(R)$ the backscatter ratio, $K$ the lidar constant, $R$ the range, and $i=\mathrm{m}$, a denotes the molecular and aerosol component, respectively. For our purposes, $\beta_{\mathrm{m}}$ and $\alpha_{\mathrm{m}}$ are known functions of height, determined either from a standard 

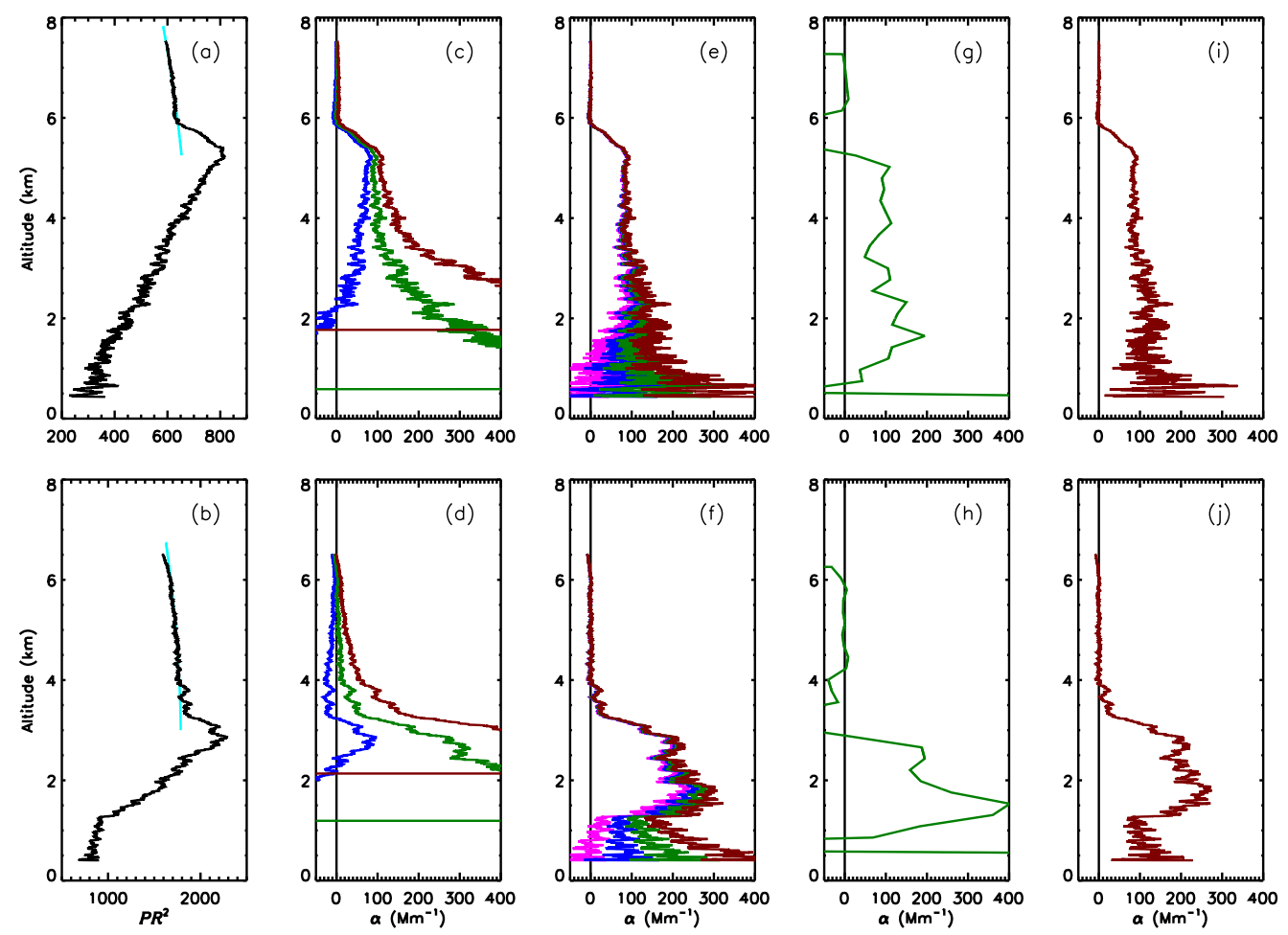

Fig. 1. (a, b) Range corrected signal profiles in arbitrary units for (a) Fennec flight B705, 12/6/2012 at 13:17 UTC, integration time 6 min $\left(23.9^{\circ} \mathrm{N}, 5^{\circ} \mathrm{W}\right)$, and (b) SAMBBA flight B741, 26/9/2012 at 15:13 UTC, integration time 1 min $\left(10.1^{\circ} \mathrm{S}, 52.6^{\circ} \mathrm{W}\right)$. The cyan line shows the fit to a Rayleigh scattering profile above the aerosols. (c, d) Aerosol extinction coefficient retrieved with the outward Fernald-Klett inversion, assuming a backscatter ratio of 0.98 (blue), 1 (green) and 1.02 (brown) for the molecular layer above the aerosol top. (e, f) Aerosol extinction coefficient retrieved with the inward Fernald-Klett inversion, assuming an average extinction coefficient of 0 (pink), 75 (blue), 150 (green), and 250 (brown) for the $0.5-1 \mathrm{~km}$ altitude range. $(\mathbf{g}, \mathbf{h})$ Aerosol extinction coefficient retrieved with the improved slope method. (i, j) Aerosol extinction coefficient retrieved with the slope-Fernald method.

atmosphere or from observations. This equation can be rewritten as follows:

$\alpha_{\mathrm{a}}=-\frac{1}{2} \frac{\mathrm{d} \ln P^{\prime}}{\mathrm{d} R}+\frac{1}{2} \frac{\mathrm{d} \ln \beta_{\mathrm{m}}}{\mathrm{d} R}+\frac{1}{2} \frac{\mathrm{d} \ln \mathrm{BR}}{\mathrm{d} R}$

where $P^{\prime}(R)=P e^{2 \int_{0}^{R} \alpha_{\mathrm{m}} \mathrm{d} R}$ can be easily computed (lidar signal corrected for molecular extinction). To be able to apply the improved slope method, we must be able to neglect the third term, i.e. assume $\mathrm{BR}=$ constant; this is equivalent to assuming that the aerosols are "well mixed" with the air molecules, as for example one can expect in a convective boundary layer. With this assumption, $\alpha_{\mathrm{a}}$ can easily be computed from the slope of $\ln P^{\prime}$ (improved slope method):

$\alpha_{\mathrm{a}} \simeq-\frac{1}{2} \frac{\mathrm{d} \ln P^{\prime}}{\mathrm{d} R}+\frac{1}{2} \frac{\mathrm{d} \ln \beta_{\mathrm{m}}}{\mathrm{d} R}$.

It has to be mentioned however that for hygroscopic aerosols, in a well-mixed but humid boundary layer, the assumption of a constant backscatter ratio will not be correct, since particles will grow towards the top of the boundary layer, where the relative humidity is larger. Note also that the second term on the right-hand side of Eq. (3) is a meteorologically determined quantity independent of the lidar signal and of the aerosol distribution; for a lidar pointing exactly at nadir it is equal to $1 / 2 H-\gamma / 2 T$, where $H$ is the scale height, $T$ the temperature, and $\gamma$ the lapse rate (it is in general equal to $40-60 \mathrm{Mm}^{-1}$ ).

Figure $1 \mathrm{~g}$ and $\mathrm{h}$ show the result of the improved slope method applied to the range-corrected signal profiles of Fig. 1a and b. Since shot noise is amplified via the signal derivative in the first term, in order to have a meaningful profile, vertical sampling has been reduced to a point every $225 \mathrm{~m}$, hence the coarser data. In Fig. 1g we observe an overall magnitude of the extinction coefficient within the aerosol layer which is compatible with its magnitude in Fig. 1e; this result was expected because in this case the Saharan dust layer appears pretty homogeneous. This is however not true at the top edge of the layer, which is not picked up correctly by the improved slope method: as the range-corrected signal at this altitude increases with range instead of decreasing, the result is a negative extinction coefficient (an unphysical result). Results in Fig. 1h are more critical, because the layer is not homogeneous $(\mathrm{BR} \neq$ constant); however the obtained 
extinction magnitude is still reasonably within $\pm 50 \%$ of the profiles in Fig. 1f, which is rather encouraging. Again, we can notice that the improved slope method is not good for identifying small-scale features and layer boundaries, as they are dominated by inhomogeneities in the aerosol distribution.

\subsection{A new approach: the slope-Fernald method}

In the previous sections we have seen that the inward Fernald-Klett with a far-range reference is quite good for determining the nearer (upper) part of the aerosol profile. Moreover, the layer boundaries and small-scale features are correctly represented into the extinction profile. At the far (lower) end of the profile, however, a large uncertainty exists on the magnitude of the aerosol extinction coefficient, because a reference value has to be "guessed". On the other hand, the improved slope method is poor at yielding a detailed aerosol vertical distribution, but it can help estimate the extinction coefficient within a layer, without requiring an a priori reference value.

In Fig. 1i and $\mathrm{j}$ a mixed approach is used: the profiles are computed with the Fernald-Klett approach, where the reference value has been set using the improved slope method; for brevity, this approach shall be denoted with the term "slopeFernald method". As shown, the slope method can give sensible results only far from an aerosol layer's edges; we therefore require the reference height interval to be set where the aerosol distribution seems to be sufficiently homogeneous. The reference height interval has been set at $0.5-1.6 \mathrm{~km}$ for Fig. $1 \mathrm{i}$ and at $0.6-1.2 \mathrm{~km}$ for Fig. $1 \mathrm{j}$, and the average extinction coefficient within that range has been used as reference to the Fernald-Klett method. The results seem to make sense, but of course in terms of solving the radiative transfer in the lidar equation, there is not a full certainty for the lowest part of the profile (below $\sim 2 \mathrm{~km}$ ). If the actual aerosol distribution did have a gradient in a place where instead homogeneity has been assumed, then the actual profiles would look like those in Fig. 1e and f, with no possibility to choose the correct one unless additional data were available. If the choice of the reference height is done intelligently, and if the uncertainty for the lower part of the profile is correctly taken into account, however, the profiles displayed in Fig. 1i and $j$ represent the best estimate that can be inferred from the lidar data alone, if no additional information is available.

\subsection{Validation for the cases under study}

The results of the slope-Fernald approach shown in Fig. 1i and $\mathrm{j}$ can be further verified using other available data. Figure $2 \mathrm{a}$ and $\mathrm{b}$ show the respective thermodynamic diagrams obtained from dropsondes nearest in time to these lidar profiles. We recall that, whereas we can trust the lidar profiles in their upper portion, the derivation of aerosol extinction coefficient for the lower part of the profiles relies on the assumption of having a homogeneous layer in the reference height interval, the latter having been chosen based on a qualitative and subjective analysis of the lidar profile.

We can see from Fig. 2a that for this case the atmosphere was unstable (dry adiabat) from the ground to $500 \mathrm{hPa}$ $(5.4 \mathrm{~km})$, and capped by a $5^{\circ} \mathrm{C}$ temperature inversion in the first sonde. This evidence shows that the atmosphere is made of two layers, each being well-mixed: a boundary layer nearer the ground and a residual layer above; see also the slightly higher dew point in the residual layer, with its base at $815 \mathrm{hPa}(1.5 \mathrm{~km})$ for the first sonde, and $770 \mathrm{hPa}(2 \mathrm{~km})$ for the second sonde. The reference height interval falls therefore within the boundary layer, and the assumption of homogeneity within it is a reasonable one. For a more detailed discussion of this particular case study in terms of atmospheric stability and dynamics, see Garcia-Carreras et al. (2013).

The tephigram in Fig. 2b shows a more complex air mass structure and history, with a temperature profile in general indicating the formation of deep convective clouds (saturated adiabat). Indeed, intense cloud cover had been observed $\sim 300 \mathrm{~km}$ to the west. In particular, the reference height interval $(910-850 \mathrm{hPa})$ corresponds to a layer where temperature follows a saturated adiabat. The air not being saturated, the layer is stable, but there are reasons to believe that it had undergone vertical mixing in a recent past. Hence again the assumption of homogeneity in this layer is compatible with the thermodynamic diagram. Note that the signature of the elevated and optically thick aerosol layer between 1.3 and $3.3 \mathrm{~km}$ (aerosol optical depth, AOD $\sim 0.4$ ) is not easily found in the tephigram.

For the first flight, additional evidence of the proposed method doing a good job is given by the comparison with in situ data, collected as the aircraft descended within the aerosol layer. The aircraft carried a three-wavelength nephelometer and a particle soot absorption photometer (PSAP); by combining these measurements one can reconstruct the aerosol extinction coefficient, which shall here for brevity be referred to as the nephelometer data, and which can be directly compared to the lidar-derived extinction coefficient. Figure $2 \mathrm{c}$ compares the profile derived by lidar with a nephelometer profile obtained during descent, showing that the two datasets agree substantially within their respective noise, especially in the lower layers. The aircraft later also performed six 10-min straight level runs at different altitudes below its previous lidar observations transect: the average and standard deviation of the nephelometer readings for each of these runs is also shown in the figure. Whereas a small discrepancy can be observed above $\sim 3 \mathrm{~km}$, this one can be explained in terms of the measurements not being simultaneous (the aircraft terminated the run at $4.25 \mathrm{~km}$ two hours after the lidar profile had been taken). Again, the extinction coefficient seems confirmed in the lower layers, and this is what is most important.

The above verifications prove that, for these two case studies, the extinction profiles obtained using the slope-Fernald approach make sense and are compatible with other available 

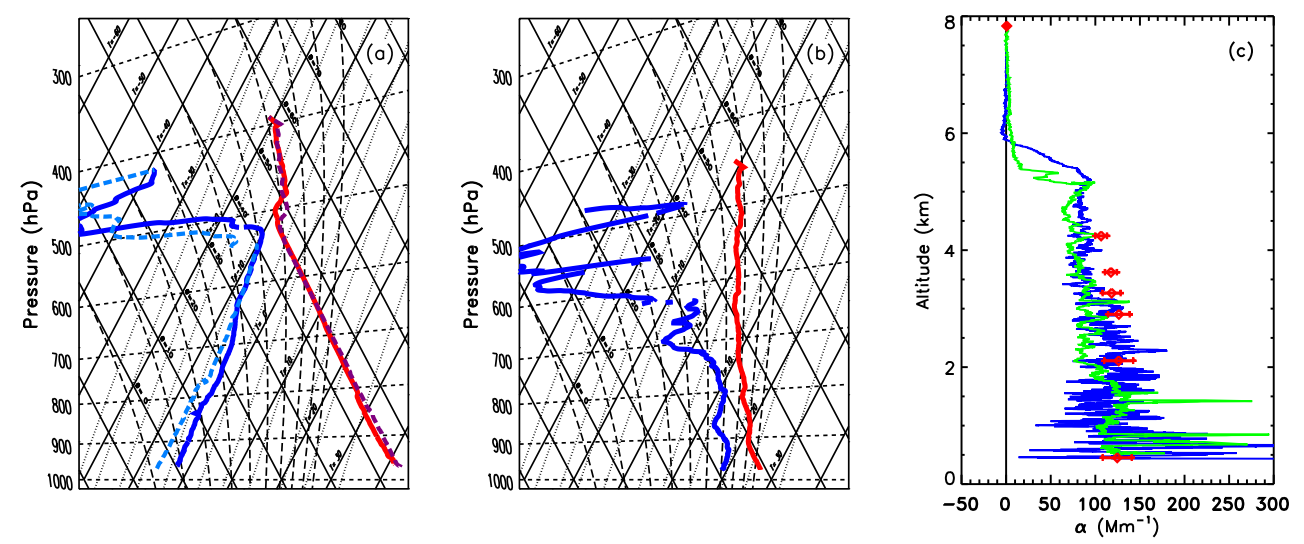

Fig. 2. (a) Tephigram derived during flight B705, from dropsondes launched at 13:13 (solid lines) and 13:20 (dashed lines): temperature (red and purple lines); dew point (dark and light blue lines). (b) Tephigram derived during flight B741 from a dropsonde launched at 15:08 (lines coloured as in a). (c) Aerosol extinction coefficient profile for flight B705: derived by lidar using the slope-Fernald approach, when flying above the aerosol layer at 13:17 (355 nm, blue line); derived by nephelometer and PSAP, during aircraft descent from 13:20 to 13:50 $(550 \mathrm{~nm}$, green line); derived by nephelometer and PSAP, during straight level runs from 13:53 to 15:13 (550 nm, red error bars).

data. This does not alter the fact that the inversion of the lidar data presents a large uncertainty in the lower part of the vertical profile; this uncertainty, however, is strongly reduced $1-2 \mathrm{~km}$ above the reference height.

\section{Exploring the advantages and limits of the method}

\subsection{Simulating lidar signals}

In this section, the advantages and limits of the slope-Fernald method are investigated, by feeding the algorithm with simulated lidar profiles. This section results from 78 simulated lidar profiles and their subsequent analysis under different assumptions (resulting in 138 different retrievals). The starting point of each simulation is a chosen aerosol extinction profile, $\alpha_{\mathrm{a}}^{0}(z)$, which we shall call the original profile or the true profile, and a lidar ratio $S^{0}$ (the true lidar ratio); based on them and on an assumed lidar constant $K$, a lidar signal profile is simulated, to which a constant background $N_{0}$ is added. The aerosol layer is assumed to be extended from ground level to $4 \mathrm{~km}$, with an extinction coefficient ranging $50-1000 \mathrm{Mm}^{-1}$ and a lidar ratio ranging $20-100 \mathrm{sr}$. The aircraft is assumed to be flying at $8 \mathrm{~km}$ and the lidar to be pointing down vertically, with a range resolution of $1.5 \mathrm{~m}$. In order to account for shot noise, a random noise is added to the lidar signal $N$ so determined, with standard deviation $\delta N=$ $B \sqrt{N}$; the shot noise can optionally be turned off, as shall be done in the initial simulations. To simulate the performance of the lidar aboard the BAe-146 aircraft in daytime, with an integration time of $1 \mathrm{~min}$, the following are assumed: a lidar constant $K=2.5 \times 10^{8} \mathrm{~m} \mathrm{sr}$, a background $N_{0}=2.5 \times 10^{-5}$, and a shot-noise factor $B=5 \times 10^{-3}$ (these quantities are approximate and instrument-dependent). The signal then undergoes the standard basic pre-processing, which includes smoothing, background subtraction, and range correction. The range-corrected signal $P$ is thus ready to be submitted to the same data analysis code that is used for actually measured data. Data analysis with the slope-Fernald method will require an assumed lidar ratio $S$ (which may be different than $S^{0}$ ) and a reference layer; its output will be the retrieved aerosol extinction coefficient $\alpha_{\mathrm{a}}(z)$, which can be directly compared to $\alpha_{\mathrm{a}}^{0}(z)$ to evaluate their agreement. A similar comparison is done between the retrieved and true AOD, $\tau$ and $\tau^{0}$, respectively, which are computed by integrating the extinction coefficient.

Before proceeding, the following basic self-consistency verifications are made (not shown here). The first verification is that the retrieved aerosol profile does actually match the original profile when the reference value to the FernaldKlett solution is set identical to the original extinction coefficient. The second verification is that the slope-Fernald approach reconstructs the aerosol profile correctly when the original profile is set for $\mathrm{BR}=$ constant (proportional to a Rayleigh scattering profile). Both verifications are based on setting $S=S^{0}$, with shot noise switched off, and are run with the whole range of extinction coefficients and lidar ratios. In both cases, profiles within $5 \mathrm{Mm}^{-1}$ and $1 \%$ of the original are retrieved, with the AOD within 0.02 and $1 \%$ of the original; we shall therefore assume that these are the expected numerical errors in the code.

\subsection{Constant aerosol profile}

In this section, the case of a constant aerosol profile from 0 to $4 \mathrm{~km}$ is investigated. Simulated lidar profiles have been obtained for all the combinations of $\alpha_{\mathrm{a}}^{0}=50,100,200,300$, $500,750,1000 \mathrm{Mm}^{-1}$ and $S^{0}=20,40,70,100 \mathrm{sr}$ (28 simulations). The reference height $Z_{\mathrm{c}}$ has been set to $500 \mathrm{~m}$ for all 
profiles, and the slope-Fernald retrievals are based on a lidar ratio matching exactly the assumed one, $S=S^{0}$.

Figure $3 \mathrm{a}$ shows an example profile, for which $\alpha_{\mathrm{a}}^{0}=200 \mathrm{Mm}^{-1}$ and $S^{0}=70 \mathrm{sr} \quad\left(\tau^{0}=0.8\right)$. The automated algorithm sets the reference value as follows: $\alpha_{\mathrm{a}}\left(Z_{\mathrm{c}}\right)=213 \mathrm{Mm}^{-1}$, thus committing an error of $+7 \%$. This error is due to the assumption of a constant BR with height near the reference height, whereas we have a constant $\alpha_{\mathrm{a}}^{0}$ instead (these two conditions being similar but not identical). However, it can be seen see that in this case the error decreases quickly as we move inward (upward): $\Delta \alpha_{\mathrm{a}}\left(Z_{\mathrm{c}}+500 \mathrm{~m}\right)=+3 \%$ and $\Delta \alpha_{\mathrm{a}}\left(Z_{\mathrm{c}}+1 \mathrm{~km}\right)=+1.5 \%$. The retrieved profile is very close to the original profile. This is very encouraging, and is reflected in terms of the retrieved AOD $\tau=0.82(+2 \%)$.

A similar evaluation has been repeated for each simulated profile, and the results are summarised in Fig. $3 \mathrm{~b}$ and $\mathrm{c}$ in absolute and in relative terms, respectively. We observe that the lower the lidar ratio, the larger the error committed. Moreover, the larger $\alpha_{\mathrm{a}}^{0}$ is, the larger the error in absolute terms, but the opposite is to be said when it is analysed in relative terms. The error remains quite reasonable, and in the worst case scenario studied here it is less than $25 \%$ at $Z_{\mathrm{c}}$. Moreover, for most cases (85\% of those discussed) at $Z_{\mathrm{c}}+1 \mathrm{~km}$ the error is reduced to less than $5 \%$. The relative error in the AOD (not shown) follows similar features to the error in $\alpha\left(Z_{\mathrm{c}}+1 \mathrm{~km}\right)$. We see also that the usefulness of the slopeFernald method is limited for low extinction coefficients and low lidar ratios, and therefore more care is recommended if one wishes to extend it to cases where $\alpha_{\mathrm{a}}\left(Z_{\mathrm{c}}\right)<50 \mathrm{Mm}^{-1}$ or $S<20$ sr.

\subsection{Oscillations in the aerosol profile}

To simulate inhomogeneity, an oscillating aerosol profile has been added to the extinction coefficient of the previous simulation; see an example in Fig. 4a. The oscillation has an amplitude of $20 \%$ of the underlying constant extinction, and has three peaks within the aerosol layer: at $0.5,2$, and $3.5 \mathrm{~km}$. The aerosol profile looks therefore like the case of an atmosphere with multiple layers. As discussed earlier, inhomogeneity at the reference height is crucial, and the slope method will give an incorrect result at layer edges, whereas it will give the best result near the layer maximum. This is exemplified in the figure: if the reference is taken at $500 \mathrm{~m}$, the retrieved profile follows quite well the original profile (blue dashed line), but if the reference height is chosen at 400 or $600 \mathrm{~m}$, i.e. where the aerosol profile is sloping (green lines), a larger error results. As in the previous examples, however, the error committed decreases as one moves inward from the reference height.

Figure $4 \mathrm{~b}$ and $\mathrm{c}$ summarise the results of similar simulations carried out for a series of different $\alpha_{\mathrm{a}}^{0}$ and $S^{0}(28$ simulated profiles and 84 retrievals). When the reference height is $Z_{\mathrm{c}}=500 \mathrm{~m}$ (solid circles), the error in $\alpha_{\mathrm{a}}\left(Z_{\mathrm{c}}\right)$ is less than
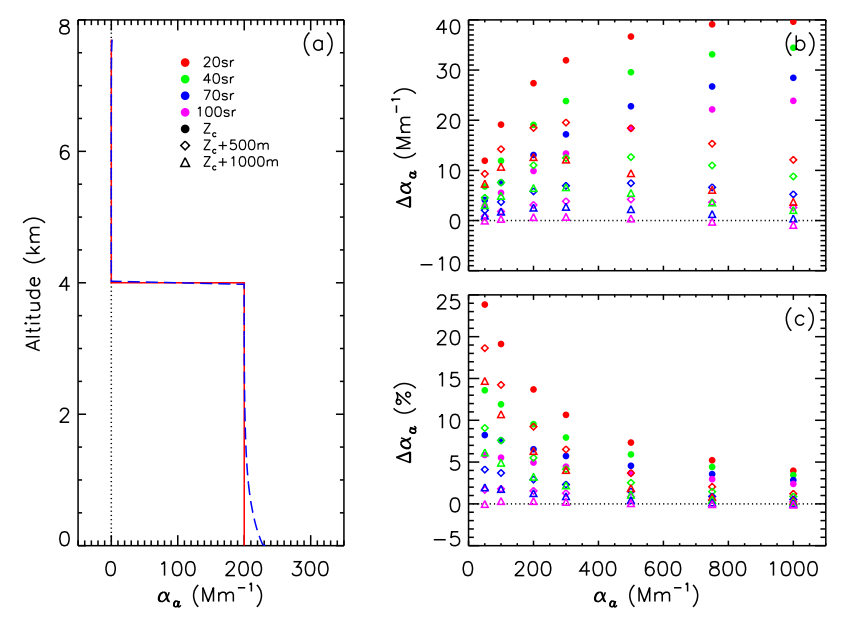

Fig. 3. Lidar signal simulation with a constant aerosol profile. (a) Red solid line: example original profile with $\alpha_{\mathrm{a}}^{0}=200 \mathrm{Mm}^{-1}$ and $S^{0}=70 \mathrm{sr}$; dashed blue line: corresponding retrieved profile obtained with the slope-Fernald method with a reference height $Z_{\mathrm{c}}=500 \mathrm{~m}$ and $S=S^{0}$. Similar simulations are carried out for several values for $\alpha_{\mathrm{a}}^{0}$ and $S^{0}$. (b, c) Difference $\Delta \alpha_{\mathrm{a}}$, between the retrieved and the original profile at given fixed altitudes, in absolute and relative terms, respectively. Different colours indicate different lidar ratios $S^{0}$ : red, $20 \mathrm{sr}$; green, $40 \mathrm{sr}$; blue, $70 \mathrm{sr}$; and magenta, 100 sr. Different symbols indicate different altitudes where $\Delta \alpha_{\mathrm{a}}$ is evaluated: solid circles, $Z_{\mathrm{c}}$; diamonds, $Z_{\mathrm{c}}+500 \mathrm{~m}$; triangles, $Z_{\mathrm{c}}+1 \mathrm{~km}$.

$25 \mathrm{Mm}^{-1}$ in absolute terms and $20 \%$ in relative terms. Much larger errors can however occur for $Z_{\mathrm{c}}=400$ (diamonds) or $600 \mathrm{Mm}^{-1}$ (triangles), and this illustrates that a good choice of $Z_{\mathrm{c}}$ is crucial for the slope-Fernald method near the reference level. As in the case discussed above, however, the error committed on $\alpha_{\mathrm{a}}\left(Z_{\mathrm{c}}+1 \mathrm{~km}\right)$ is reduced, and for most cases ( $83 \%$ of the cases studied) it is within $20 \%$. The critical cases are either those with a lidar ratio $S^{0} \leq 20$ sr or with an extinction coefficient $\alpha_{\mathrm{a}}^{0} \leq 50 \mathrm{Mm}^{-1}$, combined with an incorrect choice of $Z_{\mathrm{c}}(400$ or $600 \mathrm{~m})$, showing again that the scientist analysing lidar data must be careful when entering these low extinction, low lidar ratio regimes. The method works definitely well, with $\Delta \alpha_{\mathrm{a}}\left(Z_{\mathrm{c}}+1 \mathrm{~km}\right)<15 \%$, in all the following cases: if the reference height $Z_{\mathrm{c}}$ is chosen correctly $(500 \mathrm{~m})$; if the lidar ratio is large $\left(S^{0} \geq 70 \mathrm{sr}\right)$; or if the extinction coefficient is large $\left(\alpha_{\mathrm{a}}^{0} \geq 300 \mathrm{Mm}^{-1}\right)$.

\subsection{Effect of the lidar ratio}

When analysing experimental data, an uncertainty exists concerning which value of the lidar ratio is used in the retrievals. Figure 5 illustrates what happens if the retrievals are carried out with a lidar ratio $S$ different than the lidar ratio $S^{0}$ used to simulate the signals. As with the traditional Fernald-Klett method, the lidar ratio influences the whole of the extinction profile, and its effect does not decrease when moving inward from the reference height. The main consequence of 

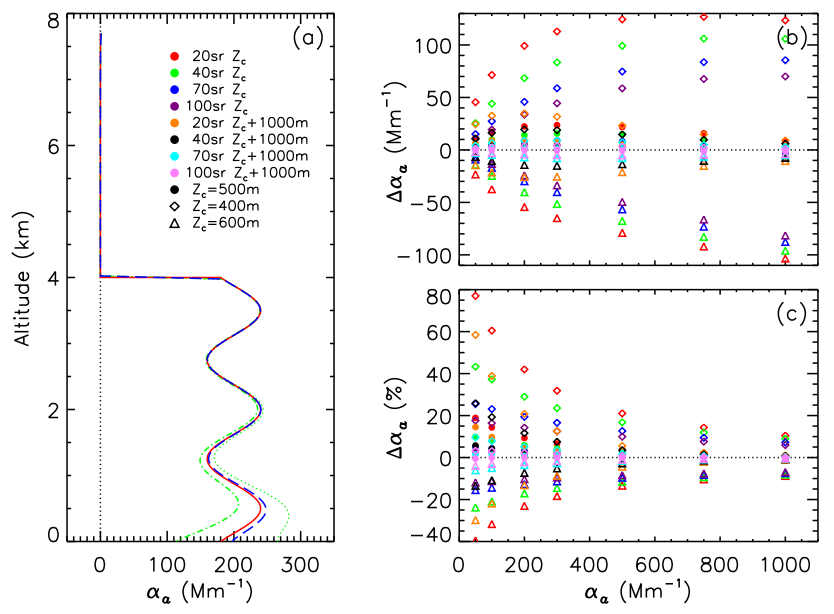

Fig. 4. Lidar signal simulation with an oscillating aerosol profile. (a) Red solid line: example original profile centred about $200 \mathrm{Mm}^{-1}$, with $S^{0}=70 \mathrm{sr}$; dashed blue line: retrieved profile with $Z_{\mathrm{c}}=500 \mathrm{~m}$ (at the oscillation maximum) and $S=S^{0}$; dotted green line: retrieved profile with $Z_{\mathrm{c}}=400 \mathrm{~m}$; dash-dotted green line: retrieved profile with $Z_{\mathrm{c}}=600 \mathrm{~m}$. (b, c) Difference $\Delta \alpha_{\mathrm{a}}$, between the retrieved and the original profile at given fixed altitudes, in absolute and relative terms, respectively. Different symbols indicate different reference heights: solid circles, $500 \mathrm{~m}$; diamonds, $400 \mathrm{~m}$; triangles, $600 \mathrm{~m}$. Different colours indicate different combinations of the lidar ratio and the altitude where $\Delta \alpha_{\mathrm{a}}$ is evaluated: red, $20 \mathrm{sr}$, $Z_{\mathrm{c}}$; green, $40 \mathrm{sr}, Z_{\mathrm{c}}$; blue, $70 \mathrm{sr}, Z_{\mathrm{c}}$; dark purple, $100 \mathrm{sr}, Z_{\mathrm{c}}$; orange, $20 \mathrm{sr}, Z_{\mathrm{c}}+1 \mathrm{~km}$; black, $40 \mathrm{sr}, Z_{\mathrm{c}}+1 \mathrm{~km}$; cyan, $70 \mathrm{sr}, Z_{\mathrm{c}}+1 \mathrm{~km}$; light purple, $100 \mathrm{sr}, Z_{\mathrm{c}}+1 \mathrm{~km}$.

changing the lidar ratio used in the retrievals is the amplification or reduction of the oscillations of the extinction coefficient, and this is mostly seen above the aerosol layer, where only using the correct lidar ratio allows reaching a null extinction coefficient. If $S$ is too small, a residual extinction coefficient is obtained above the aerosol layer, and conversely when $S$ is too large a negative (unphysical) result is obtained.

This behaviour is useful: if a molecular layer exists above the aerosols, a sanity check on $S$ can be done, and it is possible to adjust this value until the molecular layer shows correctly. Indeed, in previous papers (Marenco and Hogan, 2011; Marenco et al., 2011) two molecular layers were used, above and below an aerosol, to infer the mean lidar ratio, following the work by Di Girolamo et al. (1994). In a similar way, the lidar equation can be constrained using the slope method at the bottom of the aerosol profile, and using a traditional molecular fit at the top, to derive the lidar ratio, with a result nearly identical to $S^{0}$.

\subsection{Shot noise}

Finally, the effect of daylight entering the detector is tested. In Fig. $6 \mathrm{a}$ it is possible to see how the original profile is reconstructed when shot noise is added to the lidar signal. The effect of noise at the reference height can strongly affect the
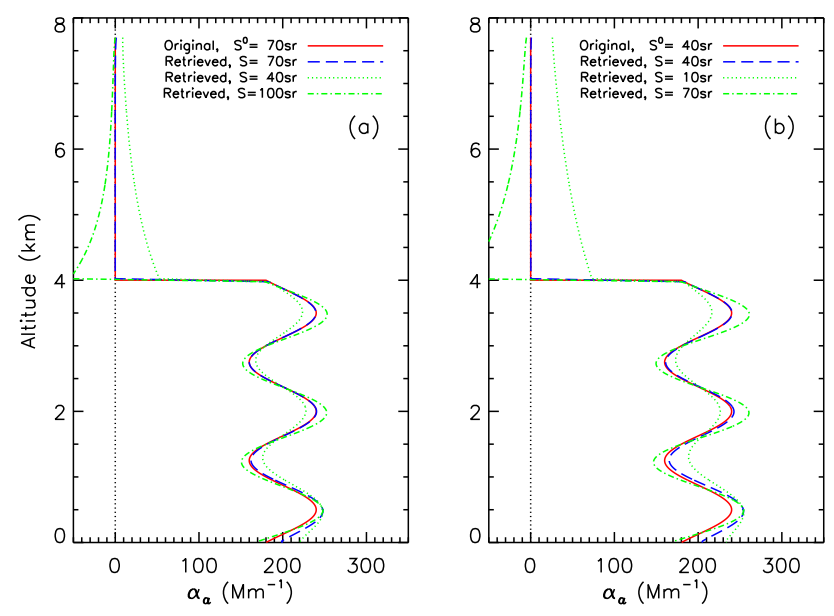

Fig. 5. Lidar signal simulation with the purpose of showing the effect of the lidar ratio $S$ used in the retrieval. (a) Original lidar ratio $S^{0}=70 \mathrm{sr}$; (b) original lidar ratio $S^{0}=40 \mathrm{sr}$. Red solid line, original profile; blue dashed line, retrieved profile with $S=S^{0}$; green dotted line, retrieved profile with $S=S^{0}-30 \mathrm{sr}$; green dash-dot line, retrieved profile with $S=S^{0}+30 \mathrm{sr}$.

retrieval, and to mitigate this risk a reference height interval has been chosen rather than a single reference height. The reference value is therefore computed for a layer $450 \mathrm{~m}$ deep, centred at $500 \mathrm{~m}$. A rather fair reconstruction of the original profile is found, although it is affected by random oscillations, as expected. The uncertainty is well represented by the amplitude of the oscillations, but no significant bias is apparent. Noise decreases moving inward from the reference height as is usual with lidar signals (more signal hits the detectors for near layers, due to attenuation along the path and the $R^{2}$ factor).

As in the previous cases, similar evaluations have been repeated for different combinations of $\alpha_{\mathrm{a}}^{0}$ and $S^{0}$, and a summary is shown in Fig. $6 \mathrm{~b}$ and c (20 simulations). The difference $\Delta \alpha_{\mathrm{a}}$ is computed for each profile for intervals centred at 1,2 and $3 \mathrm{~km}$ above the reference height. Each interval has a depth of $100 \mathrm{~m}$; again, using a layer with a given depth is necessary to avoid excessive random errors induced by shot noise on single data points. Profiles with very large aerosol optical depth $\left(\tau^{0} \geq 2\right)$ have to be regarded with suspicion, because the lidar signal transmittance through the aerosols is reduced to $2 \%$ or less. Indeed, the noise in the profiles is such that it really makes the lidar inversion a challenge. The present investigation is therefore limited to $\alpha_{\mathrm{a}}^{0} \leq 500 \mathrm{Mm}^{-1}$, which for a $4 \mathrm{~km}$ deep aerosol layer means $\tau^{0} \leq 2$

Differences between the retrieved profile and the original profile are rather large at the reference height (not shown), but as usual they decrease when moving upwards. Things start to look more acceptable for $\alpha_{\mathrm{a}}\left(Z_{\mathrm{c}}+1 \mathrm{~km}\right)$, where most points (not all, since for two cases the graphic scale is insufficient) fall within $\pm 40 \mathrm{Mm}^{-1}$ and $\pm 40 \%$ of $\alpha_{\mathrm{a}}^{0}$. At $Z_{\mathrm{c}}+2 \mathrm{~km}$, all the computed points for $\alpha_{\mathrm{a}}^{0} \leq 300 \mathrm{Mm}^{-1}\left(\tau^{0} \leq 1.2\right)$ lie 

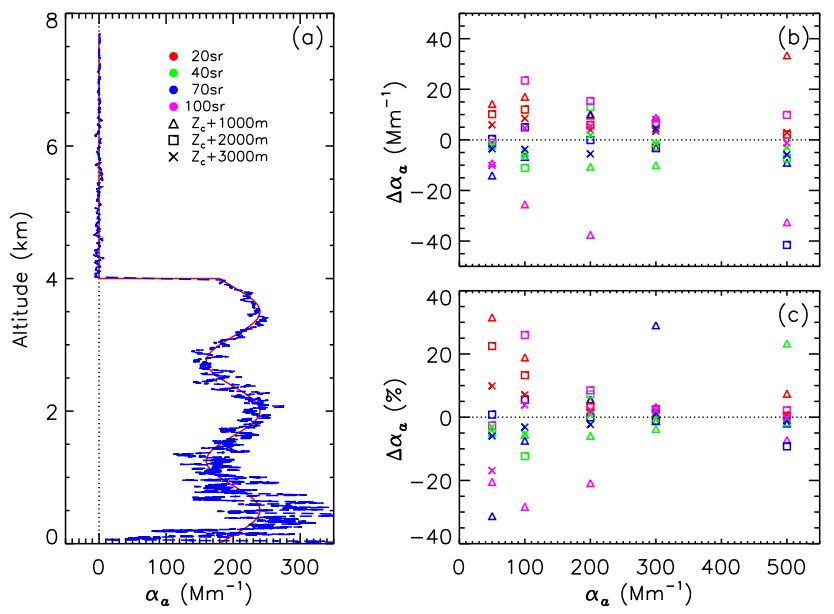

Fig. 6. Lidar signal simulation with the addition of shot noise. (a) Red solid line as in Fig. 4; dashed blue line: retrieved profile with reference taken between 275 and $725 \mathrm{~m}$ (averaged reference to reduce the influence of shot noise). (b, c) Difference $\Delta \alpha_{\mathrm{a}}$, between the retrieved and the original profile at given fixed altitudes, in absolute and relative terms, respectively. Different colours indicate different lidar ratios $S^{0}$ : red, $20 \mathrm{sr}$; green, $40 \mathrm{sr}$; blue, $70 \mathrm{sr}$; and magenta, 100 sr. Different symbols indicate different altitudes where $\Delta \alpha_{\mathrm{a}}$ is evaluated: triangles, $Z_{\mathrm{c}}+1 \mathrm{~km}$; squares, $Z_{\mathrm{c}}+2 \mathrm{~km}$; crosses, $Z_{\mathrm{c}}+3 \mathrm{~km}\left(Z_{\mathrm{c}}\right.$ being the mean reference height). All evaluations of $\Delta \alpha_{\mathrm{a}}$ are averaged over a $100 \mathrm{~m}$ deep layer centred at the indicated altitude, to reduce the influence of shot noise.

within $25 \mathrm{Mm}^{-1}$ and $30 \%$ of the original profile, but one of the cases with very large optical depth $\left(\alpha_{\mathrm{a}}^{0}=500 \mathrm{Mm}^{-1}\right.$, $\tau^{0}=2$ ) falls outside this range. All biases reduce further at $Z_{\mathrm{c}}+3 \mathrm{~km}$.

\section{Discussion}

The present research could also be useful for studies based on CALIOP data. The CALIPSO satellite was launched in 2006, and has since then provided an invaluable global dataset on the vertical structure of the atmosphere (Winker et al., 2010). Advanced algorithms have been set in place to detect automatically cloud and aerosol layers in the collected datasets, with an adaptive selection of the resolution based on return intensity, signal-to-noise ratio, and correction of the extinction due to layers above (Vaughan et al., 2009). Once layers are detected, clouds are discriminated from aerosols using thresholds in the intensity of the calibrated attenuated backscatter and the attenuated colour ratio (Liu et al., 2009). The next step is the aerosol type and lidar ratio selection (Omar et al., 2009), making use of several parameters such as the layer's integrated attenuated backscatter and depolarisation, its altitude (elevated or near-surface), and the surface type.

Once a lidar ratio is set for a layer, CALIOP extinction and backscatter profiles are computed using the Hybrid
Extinction Retrieval Algorithm (HERA), an iterative method that solves the lidar equation for a two-component atmosphere (Young and Vaughan, 2009). Regions within a scene are solved from the top of the atmosphere down to the surface. Although the analytical solution is not directly used, the algorithm can be considered basically equivalent to an outward Fernald-Klett method, on the basis that it solves the same lidar equation (except that provision is made for further improvements that may in the future account for the effects due to multiple scattering). The lidar ratio is initially selected as in Omar et al. (2009). Integration is started at the top of a layer, and rather than using normalisation to Rayleigh scattering it is based on knowledge of the lidar constant due to an accurate calibration procedure (Powell et al., 2009). As well-known, and shown again in the present paper, however, outward integration can lead to divergence of the solution in either a positive or negative direction. If this happens, in HERA the lidar ratio is adapted until a more acceptable solution is obtained; this iterative adaptation can be considered as an alternative method to seek a convergent solution, with respect to inward integration. However, the lidar ratio selection can strongly affect the retrieved aerosol profile, especially at its far end, so that when observing deep aerosol layers in principle HERA could be subject to similar or larger uncertainties to an inward integration scheme.

The inward Fernald-Klett and the slope-Fernald methods, both considered more stable methods and not requiring the iterative adaptation of the lidar ratio, could be used by scientists as an independent verification of CALIPSO datasets, starting from level 1 data as an input for single case studies and intercomparisons.

The principal message from the present paper is that inward integration of the lidar equation gives in general much better results than the outward integration (see Fernald, 1984), especially when sophisticated data analysis packages such as CALIPSO's are unavailable. In some cases, such as the nadir-viewing geometry, a problem arises in principle with the inward approach, because one cannot count on a Rayleigh scattering layer to set the reference height: the far field reference is therefore to be set within the aerosol layer, where the a priori magnitude of the extinction coefficient is uncertain. However, the effect of an error in the reference at the lower part of the aerosol layer does rapidly reduce when moving inward along the vertical profile; this makes the approach useful when layers are sufficiently deep, provided that the appropriate caveats are made.

A new method is suggested here to the data analyst, for setting the far-range reference based on the signal slope (slopeFernald approach). We believe that a critical review of the assumptions necessary for extinction profile retrieval is needed on a profile-by-profile basis, and that automated implementation of the method is not to be recommended unless further research delivers objective criteria. The reference layer will possibly be chosen as close as possible to the surface, where either the qualitative interpretation of lidar signal or 
thermodynamic considerations suggest that the atmosphere is sufficiently well-mixed (and if circumstances favouring aerosol inhomogeneities due to large relative humidity gradients are ruled out). In the best case scenario, this is a region where the signal has a constant slope, but if this is unavailable then the best is to select the reference near the aerosol maximum. In the absence of confirmation from additional data, no certainty actually exists as to how true the retrieved extinction is in the lower part of the profile, but it will represent the best estimate possible (with a large uncertainty). The bias at the reference can easily amount to $100 \%$ as shown in the example simulations where the reference is actually taken at a height where an aerosol gradient actually exists. Expert judgement and critical review of results can help reduce the bias, and moreover it is advisable to make use of dropsondes during flights so as to have a clearer picture of the thermodynamic structure and mixing state of the layers near the surface. The good news however is that the bias decreases with height, and that $1-2 \mathrm{~km}$ above the reference height the retrieved profile becomes more or less independent of assumptions.

However, one has to keep in mind the limits of the method. A fundamental one is given by the aerosol optical depth, as is always for lidar observations: low transmission will mean an unacceptable signal-to-noise ratio at the reference height. With the simulated levels of noise (similar to our instrument's in daytime), the limit seems to be at around $\tau^{0}=1.5-2$, which is a rather large optical depth. One should in any case be careful when working on cases with $\tau^{0}>1$. The optical depth limit will be dependent upon instrument specifications and amount of daylight.

The simulations also show that additional care is needed when working with low extinction coefficients (less than $\left.50 \mathrm{Mm}^{-1}\right)$ and small lidar ratios $\left(S^{0} \leq 20 \mathrm{sr}\right)$, since errors will be larger than in other cases. For as much as the lidar ratio is concerned, relatively large uncertainties on its magnitude $( \pm 30 \mathrm{sr}$ ) do not seem to have a dramatic effect on the retrievals, and moreover if a Rayleigh scattering layer exists above the aerosols, it can help constrain the lidar ratio.

All the above considerations have been drawn within the framework of single scattering. For very dense layers, a need to account for multiple scattering may arise. If a simple treatment is used such as in Platt (1979) and Young and Vaughan (2009), then the effect of multiple scattering can be parametrised by using an effective lidar ratio $S^{\prime}=\eta S$ $(\eta<1)$, and the treatment within this paper can be kept (provided that $\eta$ can be separately evaluated).

From the above simulations, the systematic errors that the method introduces can be approximately rated at $\pm 100 \%$ at the reference height, $\pm 40 \mathrm{Mm}^{-1}$ and $\pm 40 \%$ in the 1$2 \mathrm{~km}$ above it, and $\pm 10 \mathrm{Mm}^{-1}$ and $\pm 20 \%$ at higher altitudes. Whereas the inversion assumptions (reference height) directly drive the error at the reference height itself, shot noise seems to be at least equally responsible for errors above $Z_{\mathrm{c}}+1 \mathrm{~km}$.
Acknowledgements. Airborne data were obtained using the BAe-146-301 Atmospheric Research Aircraft (ARA) flown by Directflight Ltd and managed by the Facility for Airborne Atmospheric Measurements (FAAM), which is a joint entity of the Natural Environment Research Council (NERC) and the Met Office. Fennec was funded by NERC (grant NE/G017166/). SAMBBA was funded by the Met Office and NERC (grant NE/J009822/1). This research has been carried partly at the University of Leeds, within the framework of the Met Office Academic Partnership scheme.

Edited by: V. Amiridis

\section{References}

Amiridis, V., Wandinger, U., Marinou, E., Giannakaki, E., Tsekeri, A., Basart, S., Kazadzis, S., Gkikas, A., Taylor, M., Baldasano, J., and Ansmann, A.: Optimizing Saharan dust CALIPSO retrievals, Atmos. Chem. Phys. Discuss., 13, 14749-14795, doi:10.5194/acpd-13-14749-2013, 2013.

Angelo, C.: Amazon fire analysis hits new heights, Nature News, 59, 338-349, doi:10.1038/nature.2012.11467, 2012.

Burton, S. P., Ferrare, R. A., Vaughan, M. A., Omar, A. H., Rogers, R. R., Hostetler, C. A., and Hair, J. W.: Aerosol classification from airborne HSRL and comparisons with the CALIPSO vertical feature mask, Atmos. Meas. Tech., 6, 13971412, doi:10.5194/amt-6-1397-2013, 2013.

Collis, R. T. H.: Lidar: a new atmospheric probe, Q. J. Roy. Meteorol. Soc., 92, 220-230, 1966.

Di Girolamo, P., Cacciani, M., Sarra, A. D., Fiocco, G., and Fuà, D.: Lidar observations of the Pinatubo aerosol layer at Thule, Greenland, Geophys. Res. Lett., 21, 1295-1298, 1994.

Donovan, D. P. and Apituley, A.: Practical depolarization-ratiobased inversion procedure: lidar measurements of the Eyjafjallajökull ash cloud over the Netherlands, Appl. Optics, 52, 2394 2415, 2013.

Fernald, F. G.: Analysis of atmospheric lidar observations: some comments, Appl. Optics, 23, 652-653, 1984.

Fernald, F. G., Herman, B. M., and Reagan, J. A.: Determination of Aerosol Height Distribution by Lidar, J. Appl. Meteorol., 11, 482-489, 1972.

Ferrare, R., Melfi, S., Whiteman, D., Evans, K., and Leifer, R.: Raman lidar measurements of aerosol extinction and backscattering. 1. Methods and comparisons, J. Geophys. Res., 103, 1966319672, 1998.

Garcia-Carreras, L., Parker, D. J., Marsham, J. H., Rosenberg, P. D., Marenco, F., and Mcquaid, J. B.: The Saharan atmospheric boundary layer: Turbulence, stratification and mixing, Geophys. Res. Abstr., EGU2013-9850, EGU General Assembly 2013, Vienna, Austria, 2013.

Groß, S., Freudenthaler, V., Wiegner, M., Gasteiger, J., Geiß, A., and Schnell, F.: Dual-wavelength linear depolarization ratio of volcanic aerosols: Lidar measurements of the Eyjafjallajökull plume over Maisach, Germany, Atmos. Environ., 48, 85-96, 2012.

Groß, S., Esselborn, M., Weinzierl, B., Wirth, M., Fix, A., and Petzold, A.: Aerosol classification by airborne high spectral resolution lidar observations, Atmos. Chem. Phys., 13, 2487-2505, doi:10.5194/acp-13-2487-2013, 2013. 
Ingmann, P.: EarthCARE - Earth Clouds, Aerosols, and Radiation Explorer, Vol. Sp-1279, ESA Tech. Rep., 2004.

Klett, J. D.: Lidar inversion with variable backscatter/extinction ratios, Appl. Optics, 24, 1638-1643, 1985.

Kovalev, V. A.: Lidar measurements of the vertical aerosol extinction profiles with range-dependent backscatter-to-extinction ratios, Appl. Optics, 32, 6053-6065, 1993.

Kunz, G. J. and de Leeuw, G.: Inversion of lidar signals with the slope method, Appl. Optics, 32, 3249-3256, 1993.

Liu, Z., Vaughan, M., Winker, D., Kittaka, C., Getzewich, B., Kuehn, R., Omar, A., Powell, K., Trepte, C., and Hostetler, C.: The CALIPSO lidar cloud and aerosol discrimination: Version 2 algorithm and initial assessment of performance, J. Atmos. Ocean. Technol., 26, 1198-1213, 2009.

Marenco, F. and Hogan, R. J.: Determining the contribution of volcanic ash and boundary-layer aerosol in backscatter lidar returns: a three-component atmosphere approach, J. Geophys. Res., 116, D00U06, doi:10.1029/2010JD015415, 2011.

Marenco, F., Santacesaria, V., Bais, A. F., Balis, D., di Sarra, A., Papayannis, A., and Zerefos, C.: Optical properties of tropospheric aerosols determined by lidar and spectrophotometric measurements (Photochemical Activity and Solar Ultraviolet Radiation campaign), Appl. Optics, 36, 6875-6886, 1997.

Marenco, F., Johnson, B., Turnbull, K., Newman, S., Haywood, J., Webster, H., and Ricketts, H.: Airborne Lidar Observations of the 2010 Eyjafjallajökull Volcanic Ash Plume, J. Geophys. Res., 116, D00U05, doi:10.1029/2011JD016396, 2011.

Omar, A. H., Winker, D. M., Kittaka, C., Vaughan, M. A., Liu, Z., Hu, Y., Trepte, C. R., Rogers, R. R., Ferrare, R. A., Lee, K.-P., Kuehn, R. E., and Hostetler, C. A.: The CALIPSO Automated Aerosol Classification and Lidar Ratio Selection Algorithm, J. Atmos. Ocean. Technol., 26, 1994-2014, 2009.

Platt, C. M. R.: Remote sounding of high clouds: I. Calculation of visible and infrared optical properties for lidar and radiometer measurements, J. Appl. Meteorl., 18, 1130-1143, 1979.
Powell, K. A., Hostetler, C. A., Liu, Z., Vaughan, M. A., Kuehn, R. E., Hunt, W. H., Lee, K.-P., Trepte, C. R., Rogers, R. R., Young, S. A., and Winker, D. M.: CALIPSO lidar calibration algorithms. Part I: Nighttime 532-nm parallel channel and 532nm perpendicular channel, J. Atmos. Ocean. Technol., 26, 20152033, 2009.

Ryder, C. L., Highwood, E. J., Rosenberg, P. D., Trembath, J., Brooke, J. K., Bart, M., Dean, A., Crosier, J., Dorsey, J., Brindley, H., Banks, J., Marsham, J. H., McQuaid, J. B., Sodemann, H., and Washington, R.: Optical properties of Saharan dust aerosol and contribution from the coarse mode as measured during the Fennec 2011 aircraft campaign, Atmos. Chem. Phys., 13, 303325, doi:10.5194/acp-13-303-2013, 2013.

Takamura, T., Sasanu, Y., and Hayasaka, T.: Tropospheric aerosol optical properties derived from lidar, sun photometer, and optical particle counter measurements, Appl. Optics, 33, 7132-7140, 1994.

Vaughan, M. A., Powell, K. A., Kuehn, R. E., Young, S. A., Winker, D. M., Hostetler, C., Hunt, W. H., Liu, Z., McGill, M. J., and Getzewich, B. J.: Fully automated detection of cloud and aerosol layers in the CALIPSO lidar measurements, J. Atmos. Ocean. Technol., 26, 2034-2050, 2009.

Winker, D. M., Pelon, J., Coakley, Jr., J. A., Ackerman, S. A., Charlson, R. J., Colargo, P. R., Flamant, P., Fu, Q., Hoff, R. M., Kittaka, C., Kubar, T. L., Le Treut, H., McCormick, M. P., Mégie, G., Poole, L., Powell, K., Trepte, C., Vaughan, M. A., and Wielicki, B. A.: The CALIPSO mission: A Global 3D View of Aerosols and Clouds, B. Am. Meteorol. Soc., 91, 1211-1228, 2010.

Young, S. A. and Vaughan, M. A.: The retrieval of profiles of particulate extinction from Cloud-Aerosol Lidar Infrared Pathfinder Satellite Observations (CALIPSO) data: Algorithm description, J. Atmos. Ocean. Technol., 26, 1105-1119, 2009. 